\title{
NR2A-NMDA Receptor Blockade Reverses the Lack of Morphine Analgesia Without Affecting Chronic Pain Status in a Fibromyalgia-Like Mouse Mode ${ }^{[\mathbb{S}}$
}

\author{
(D)Hiroyuki Neyama, ${ }^{1}$ Naoki Dozono, ${ }^{2}$ and Hiroshi Ueda ${ }^{2}$ \\ Department of Pharmacology and Therapeutic Innovation, Nagasaki University Institute of Biomedical Sciences, Nagasaki, Japan \\ Received September 11, 2019; accepted January 13, 2020
}

\begin{abstract}
We have developed an experimental fibromyalgia-like mouse model using intermittent cold stress (ICS), where chronic pain is generalized, female predominant, and abolished in type 1 lysophosphatidic acid receptor-knockout $\left(\mathrm{LPA}_{1}{ }^{-1-}\right.$ ) mice but is not reversed by systemic or brain treatment with morphine. We investigated two issues in the present study: (1) whether chronic pain mechanisms and lack of brain morphine analgesia are associated in the ICS model and (2) what mechanisms are involved in the lack of morphine analgesia. ICS-induced hyperalgesia was not affected in $\mu$-opioid receptor-knockout (MOPr $\left.{ }^{-/}\right)$mice, whereas the lack of brain morphine analgesia remained unchanged in $\mathrm{LPA}_{1}{ }^{--}$mice, which completely abolished the hyperalgesia in the ICS model. In contrast, the lack of morphine analgesia was abolished in NR2A-NMDA receptor-knockout $\left(\mathrm{NR}^{-\mathrm{A}^{-1}}{ }^{-1}\right.$ ) mice and blocked by intracerebroventricular (i.c.v.) injection of (R)-CPP, an NR2A antagonist, or by microinjection of siRNA NR2A into the periaqueductal gray matter region, whereas no change was observed with Ro 04-5595, an NMDA receptor subtype $2 \mathrm{~B}$ antagonist (i.c.v.). The lack of morphine analgesia was also reversed by concomitant treatment with $1 \mathrm{mg} / \mathrm{kg}$ intraperitoneal (i.p.) of dextromethorphan, which possesses
\end{abstract}

NMDA receptor antagonist activity but no analgesic activity. Finally, the hyperalgesia was completely reversed by methadone, which possesses both MOPr agonist and NMDA receptor antagonist activity. Indeed, methadone analgesia was abolished in $\mathrm{MOPr}^{-1-}$ mice. These results suggest that chronic pain status and lack of morphine analgesia are independent of each other, and that lack of morphine analgesia is mediated by activation of the NR2A-NMDA receptor system.

\section{SIGNIFICANCE STATEMENT}

This study reports that a type of intermittently repeated stress causes widespread pain that does not respond to morphine. Because this lack of morphine analgesia is not affected in mice, in which chronic pain is abolished, the mechanisms underlying chronic pain and lack of morphine analgesia are independent of each other. Through speculation that a lack of morphine analgesia may be a secondary event to endogenous opioid analgesic tolerance, the authors demonstrate that an antiopioid $N$-methyl- $D$-aspartate receptor system counterbalances the $\mu$-opioid receptor-mediated analgesic mechanisms in the intermittent cold stress model.

\section{Introduction}

Opioids are essential medicines for terminal cancer pain and are also used to treat neuropathic pain. However, a recent systematic review (Cooper et al., 2017) summarized that there

This work was supported in part by Grants-in-Aid for the Platform for Drug Discovery, Informatics, and Structural Life Science [16am0101012j0005] (H.U.) from the Japan Agency for Medical Research and Development, Japan; KAKENHI JP17H01586 (H.U.) and JP26253077 (H.U.) from JSPS.

${ }^{1}$ Current affiliation: RIKEN Center for Biosystems Dynamics Research, Kobe, Japan.

${ }^{2}$ Current affiliation: Department of Molecular Pharmacology, Kyoto University Graduate School of Pharmaceutical Sciences, Sakyo-ku, Kyoto, Japan. https://doi.org/10.1124/jpet.119.262642.

S This article has supplemental material available at jpet.aspetjournals.org. is severely limited evidence to support morphine being beneficial for neuropathic pain. The limited effectiveness of opioids in neuropathic pain means it requires high treatment doses, which may be one of the causes of the current opioid epidemic/crisis in the United States (Häuser et al., 2017). Although it remains to be fully clarified why opioids are less effective in neuropathic pain (Rashid et al., 2004; MartínezNavarro et al., 2019), we previously reported a marked reduction in $\mu$-opioid receptor (MOPr) expression in the dorsal root ganglion as one of the possible mechanisms in a mouse model with partial sciatic nerve ligation (Yamamoto et al., 2008; Uchida et al., 2010). In these studies, peripheral morphine analgesia was lost due to epigenetic silencing of $\mu$-opioid receptor (MOPr) expression, whereas brain morphine

ABBREVIATIONS: aCSF, artificial cerebrospinal fluid; BDNF, brain derived neurotrophic factor (R)-CPP 3-[(R)-2Carboxypiperazin-4-yl]-propyl-1phosphonic acid; DAMGO, [D-Ala ${ }^{2}, \mathrm{~N}-\mathrm{MePhe}^{4}$, Gly-ol]-enkephalin; DR Dorsal raphe nucleus DRD Dorsal raphe nucleus, dorsal part DRV Dorsal raphe nucleus, ventral part DRVL Dorsal raphe nucleus, ventrolateral part DMSO, dimethyl sulfoxide; DXM, dextromethorphan; EPW, electrical stimulation-induced paw withdrawal; FM, fibromyalgia; ICS, intermittent cold stress; i.c.v., intracerebroventricular; i.p., intraperitoneal; i.pl., intraplanter; IPS, intermittent psychological stress; i.t., intrathecal; $\mathrm{LPA}_{1}{ }^{-/}$, lysophosphatidic acid receptor-knockout; MOPr, $\mu$-opioid receptor; NMDA, $N$-methyl- $D$-aspartate; NR1 NMDA recepotor 1 NR2A, NMDA receptor subtype 2A; NR2B, NMDA receptor subtype 2B; P, post stress day; PAG, periaqueductal gray matter; PSD95, postsynaptic density 95; RPH3A, rabphiline 3A; s.c., subcutaneous; siNR2A, small interfering NR2A; siRNA, small interfering RNA; SSR, SSR149415; VLPAG, ventrolateral PAG; VTA, ventral tegmental area; WT, wild type. 
analgesia remained unchanged (Rashid et al., 2004; Uchida et al., 2010).

One of the representative cases of morphine inactivity is observed in fibromyalgia (FM). Harris et al. (2007) and Schrepf et al. (2016) reported that the opioid receptor binding and opioid-related functional connectivity in the cingulate cortex of patients with FM is negatively related to the degree of pain score. Because FM is currently treated with antiepileptics (pregabalin) or antidepressants (duloxetine, milnacipran) that are limited in their clinical use due to their side effects (Clauw, 2014), we started studying the molecular mechanisms underlying opioid inactivity in FM-like generalized pain models. Several experimental FM-like generalized pain disease models have been reported, which mainly use repeated or intermittent stress (DeSantana et al., 2013). We have previously developed two types of intermittent cold stress (ICS)- or intermittent psychological stress (IPS)induced FM-like generalized pain disease models, which show long-lasting generalized pain, such as somatic thermal, mechanical, chemical, and electrical pain and muscular mechanical pain (Nishiyori and Ueda, 2008; Ueda and Neyama, 2017), as observed in other generalized pain disease models (Sluka et al., 2001; Khasar et al., 2003, 2005; Nagakura et al., 2009). Mice subjected to ICS or IPS show a female-predominant pain, particularly after gonadectomy (Nishiyori and Ueda, 2008; Ueda and Neyama, 2017). In addition to the pathophysiology, these mouse models show pharmacotherapeutic similarities to clinical features seen in patients with FM (Nishiyori and Ueda, 2008; Ueda and Neyama, 2017) with pharmacological studies showing that potent antihyperalgesic effects were seen with gabapentinoids, duloxetine, and other antidepressants (Yokoyama et al., 2007; Nishiyori and Ueda, 2008; Nishiyori et al., 2011; Ueda and Neyama, 2017), in comparison with a nonsteroidal antiinflammatory drug (diclofenac) and morphine (Nishiyori et al., 2010; Ueda and Neyama, 2017), which did not show such effects. In the present study, we studied the relationship between chronic pain status and lack of morphine analgesia using an experimental FM-like model to better understand the appropriate use of opioids in chronic pain.

\section{Materials and Methods}

Animals. Male C57BL/6J mice (15-25 g) were purchased from TEXAM (Nagasaki, Japan). $\mu$ opioid receptor gene-deficient $\left(\mathrm{MOPr}^{-/-}\right)$ mice were obtained from Brigitte Kieffer (McGill Univ. Douglas Institute, Montreal, Canada) and backcrossed to the inbred C57BL/ $6 \mathrm{~J}$ mice for at least 10 generations, as reported previously (Nagai et al., 2007). Type 1 Lysophosphatidic acid receptor-knockout ( $\left.\mathrm{LPA}_{1}{ }^{-{ }^{-}}\right)$ mice were obtained from Jerold Chun (Sanford Burnham Prebys Medical Discovery Institute, La Jolla, CA), and NMDA receptor subunit NR2A gene-deficient (NR2A ${ }^{-1}$ ) mice, which have a highly homogeneous genetic background to the C57BL/6J strain (Sakimura et al., 1995), were obtained from RIKEN BRI (Wako, Japan). Animals were housed in a room maintained at $22 \% \pm 3 \%$ and $55 \% \pm 5 \%$ relative humidity with a 12-hour light/dark cycle (light from 8:00 AM to 8:00 PM) and had free access to a standard laboratory diet and tap water. Because body weights of the mice used varied by up to $20 \%$ in each set of experiments, we did not perform randomization. All experiments were carried out blind. All procedures used in this work were approved by the Nagasaki University Animal Care Committee (number: 1607201325-8) and complied with the recommendations of the International Association for the Study of Pain (Zimmermann, 1983).
All studies using animals were reported in accordance with the Animal Research: Reporting of In Vivo Experiments (ARRIVE) guideline (Kilkenny et al., 2010; McGrath et al., 2010; McGrath and Lilley, 2015).

Drugs. Drugs were administrated through i.p. $(100 \mu \mathrm{l} / 10 \mathrm{~g})$, subcutaneous (s.c., $100 \mu \mathrm{l} / 10 \mathrm{~g}$ ), i.c.v. $(5 \mu \mathrm{l})$, intrathecal (i.t., $5 \mu \mathrm{l}$ ), and intraplanter (i.pl., $20 \mu \mathrm{l}$ ) routes. Morphine hydrochloride (Takeda Chemical Industries, Osaka, Japan) was dissolved in artificial cerebrospinal fluid (aCSF; $125 \mathrm{mM} \mathrm{NaCl}, 3.8 \mathrm{mM}, \mathrm{KCl}, 1.2 \mathrm{mM}$ $\mathrm{KH}_{2} \mathrm{PO}_{4}, 26 \mathrm{mM} \mathrm{NaHCO} 3,10 \mathrm{mM}$ glucose, $\mathrm{pH}$ 7.4) for i.c.v., i.t., and i.pl. injection. 3-[(R)-2Carboxypiperazin-4-yl]-propyl-1-phosphonic acid [(R)-CPP; Funakoshi Co., Ltd., Tokyo, Japan] and 1-[2-(4Chlorophenyl) ethyl]-1,2,3,4-tetrahydro-6-methoxy-7-isoquinolinol hydrochloride (Ro 04-5595 hydrochloride; Funakoshi Co., Ltd.) were dissolved in aCSF with $0.5 \%$ dimethyl sulfoxide (DMSO) for i.c.v. injection. Dextromethorphan hydrobromide monohydrate (WAKO, Osaka, Japan) and D,L-methadone hydrochloride (Mallinckrodt Inc, St Louis, MO) were dissolved in physiologic saline with $0.5 \%$ DMSO and physiologic saline alone for i.p. and s.c. injection, respectively. Pregabalin (Sigma Aldrich, St Louis, MO) was dissolved in aCSF for i.c.v. injection. SSR149415 (SSR), a gift from Prof. Takaaki Koshimizu (Jichi Medical University, Japan), was dissolved with 1\% DMSO in aCSF for i.c.v. injection. [D-Ala ${ }^{2}, \mathrm{~N}-\mathrm{MePhe}^{4}$, Gly-ol]-enkephalin (DAMGO; Sigma Aldrich) was dissolved in aCSF for i.c.v. injection.

siRNA. The in vivo siRNA delivery using JetSI (Polyplus Transfection, France) was performed as previously reported (Guissouma et al., 2006). To confirm the brain locus of microinjected siRNA $(0.1 \mu \mathrm{g} /$ $\mu \mathrm{l}), 0.2 \%$ of Evans blue was added to the final solution. In the present study, we used three sets of antisense siRNAs or sense siRNAs (Sigma Aldrich) with the following sequences: Sense (control) siNR2A: 5'-rGUUUrAUUrCrCUrGrCrArGrGUUrCrATT-3', 5'rCrArAUrGrCrArGrAUrArCUrGUrCrGrAUTT-3' and 5'-rGrCrArArAUrCrArArGrCUUrCrArGUrCrATT-3'. Antisense siNR2A: 5'UrGrArArCrCUrGrCrArGrGrArAUrArArArCTT-3', 5'-rAUrCrGrArCrArGUrAUrCUrGrCrAUUrGTT-3', and 5'-UrGrArCUrGrArArGrCUUrGrAUUUrGrCTT-3'

Nociception Tests. The thermal pain threshold (Hargreaves test) was evaluated by the latency of paw withdrawal upon thermal stimulus (Hargreaves et al., 1988; Ueda and Neyama, 2017). Unanesthetized animals were placed in plexiglass cages on top of a glass sheet, and an adaptation period of 1-3 hour was allowed. The thermal stimulator (IITC Inc., Woodland Hills, CA) was positioned under the glass sheet and the focus of the projection bulb was aimed exactly at the middle of the plantar surface of the animal. A mirror attached to the stimulator permitted visualization of the plantar surface. In this apparatus, the regulator was set at intensity 20 , which increases the plantar surface temperature to $45.1^{\circ} \mathrm{C} \pm 1.3^{\circ} \mathrm{C}(n=10)$ at 10 seconds after the start of thermal stimulation. A cutoff time of 20 seconds was set to prevent tissue damage. A mechanical pain stimulus was then delivered to the middle of the plantar surface of the right hind paw using an Electronic von Frey Anesthesiometer with Rigid Tips (Model 2390, $90 \mathrm{~g}$ probe $0.8 \mathrm{~mm}$ in outer diameter; IITC Inc.). The pressure needed to induce a flexor response was defined as the pain threshold. An electrical stimulation-induced paw withdrawal test (EPW) was performed as described previously (Matsumoto et al., 2008; Ueda, 2017). Briefly, electrodes of a Neurometer Current Perception Threshold/C (CPT/C; Neurotron Inc., Baltimore, MD) were fastened to the planter and the insteps of the hind paw. Transcutaneous nerve stimuli with each of the three sine-wave pulses $(5,250$, and $2000 \mathrm{~Hz}$ ) were applied. The minimum intensity (microampere) at which each mouse withdrew its paw was defined as the current threshold. A mechanical muscle pain test was performed as described previously (Ueda and Neyama, 2017). Briefly, the mechanical muscle pain test was performed using a Randall-Selitto-type pressure analgesia meter MK-201D (Muromachi Kikai, Tokyo, Japan), which displays the pressure level. To evaluate the muscle pain threshold, pressure was applied to the right femoral muscle, and the threshold at which struggling behavior was observed was measured. 
ICS Model. Mice were exposed to ICS as described previously (Nishiyori and Ueda, 2008). Briefly, mice were placed in a cold room at $4^{\circ} \mathrm{C}$ overnight (from 4:30 PM to 10:00 AM), followed by alternating environmental temperatures between $24^{\circ} \mathrm{C}$ and $4^{\circ} \mathrm{C}$ every 30 minutes from $10: 00$ $\mathrm{AM}$ to $4: 30 \mathrm{PM}$ and placed in a cold room at $4^{\circ} \mathrm{C}$ overnight. These procedures were repeated twice. After the second session of alternating temperature and overnight cold stress [post stress day $(\mathrm{P}) 1$ ], the mice were returned and adapted to the room at $24^{\circ} \mathrm{C}$ for 1 hour before the behavior tests. Mice in the control group were kept at $24^{\circ} \mathrm{C}$ from 4:30 PM on the 1st day to 10:00 AM on the 4th day. During the stress period, two mice were kept in each cage $(12 \times 15 \times 10.5 \mathrm{~cm})$, with free access to food and agar in place of fluid to prevent mice being wet by water leakage from the bottle.

Stereotaxic In Vivo Microinjection. Stereotaxic microinjection into the periaqueductal gray matter (PAG) and ventral tegmental area (VTA) at P3 was performed as previously described (Tanaka et al., 2012). The RNA free water solution containing siRNA in $500 \mathrm{nl}$ was microinjected per brain locus bilaterally to the ventrolateral PAG (VLPAG) and VTA at a speed of $0.5 \mu \mathrm{l} / \mathrm{min}$ using a glass micropipette with a PN-30 micropipette puller (NARISHIGE, Tokyo, Japan) under anesthesia with pentobarbital (Nacalai Tesque, Kyoto, Japan)
$50 \mathrm{mg} / \mathrm{kg}$, i.p. Stereotaxic coordinates targeted to the VLPAG and VTA were: -4.6 and $-3.28 \mathrm{~mm}$ anterior from the Bregma, \pm 0.5 and $\pm 0.25 \mathrm{~mm}$ lateral from the midline and 2.25 and $4.5 \mathrm{~mm}$ ventral from the brain surface at the Bregma according to a mouse brain atlas (Paxions and Franklin, 2001). Immediately after the behavioral test (P5, 2 days after the microinjection), mice were sacrificed, the isolated brain dissected, and a visual assessment of the exact loci of delivered siRNA with Evans blue (0.2\%) carried out.

Real-Time Quantitative Reverse Transcription Polymerase Chain Reaction. Real-time quantitative reverse transcriptions polymerase chain reaction was performed as previously described (Ueda et al., 2018a). Briefly, Total RNA was prepared using brain tissue of VLPAG (Paxions and Franklin, 2001) from Bregma -4.04 to $-4.96 \mathrm{~mm}$ ) using TRizol Reagent (Thermo Fisher Scientific, Waltham, MA). Total RNA was converted into first-strand cDNA using a PrimeScript RT Reagent Kit (Takara Bio Inc., Kusatsu, Japan). After purification, cDNA was measured spectrophotometrically using NANODROP LITE (Thermo Fisher Scientific). The cycling parameters were 10 minutes at $95^{\circ} \mathrm{C}$ followed by 45 cycles of 15 seconds at $95^{\circ} \mathrm{C}$ and 1 minute at $60^{\circ} \mathrm{C}$. Primer sets used for Real-time qPCR
Mechanical paw pressure test

A

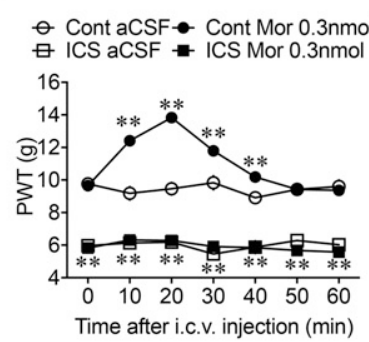

\section{B}

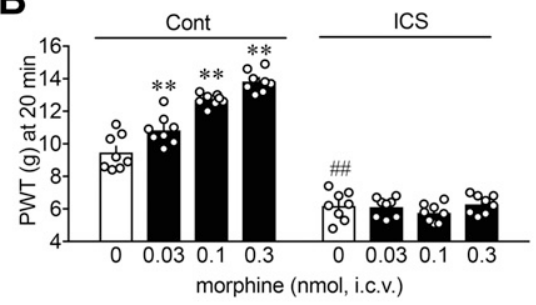

Electrical stimulation-induced paw withdrawal (EPW) test

D
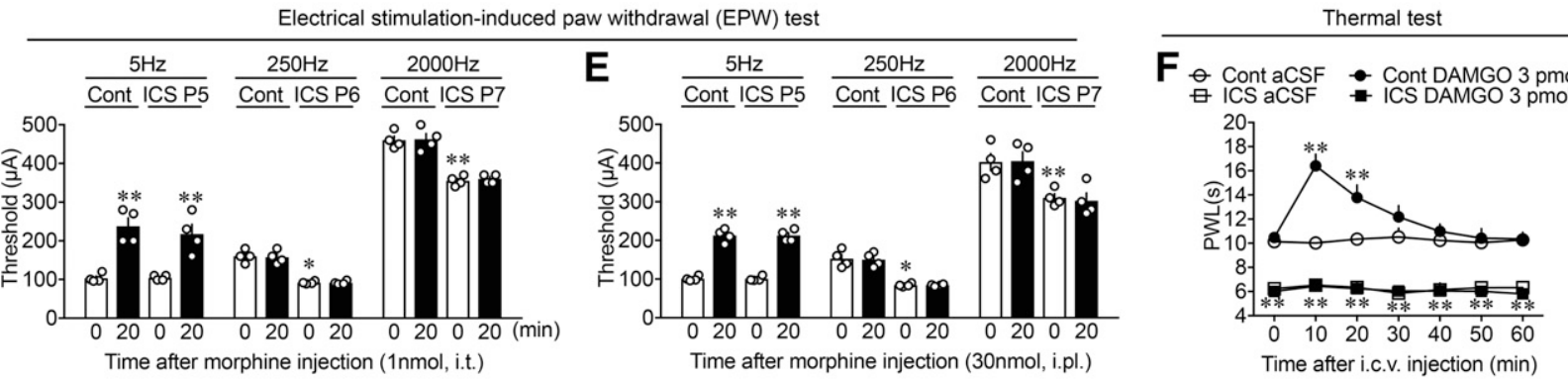

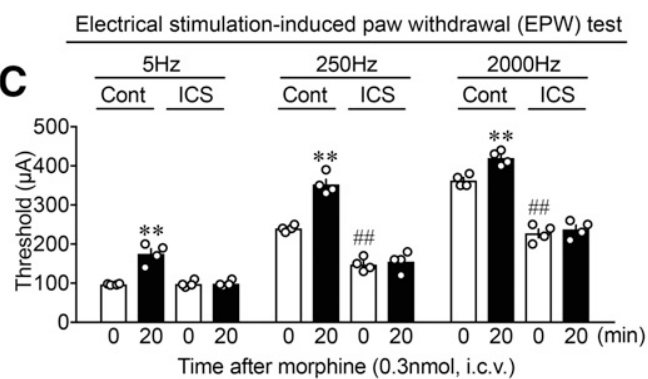

Time after morphine $(0.3 \mathrm{nmol}$, i.c.v. $)$

G

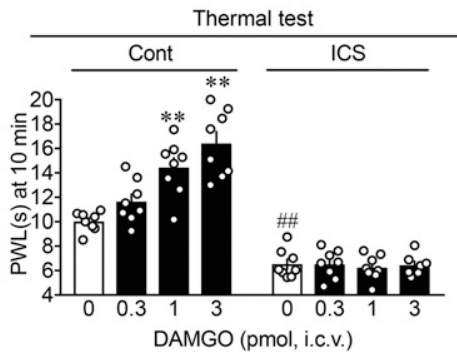

Fig. 1. Lack of analgesia by morphine and DAMGO in ICS-treated mice. (A and B) Time course (A) of morphine analgesia at $0.3 \mathrm{nmol}$ (i.c.v.) and dosedependency (B) at $0.03-0.3 \mathrm{nmol}$ (i.c.v.) at 20 minutes in control and ICS mice in the mechanical paw pressure test $(n=8)$. The morphine treatments at 0 , $0.03,0.1$, and $0.3 \mathrm{nmol}$ (i.c.v.) were consecutively performed at P5, P6, P7, and P8, respectively. (C and E) Lack of sensory fiber-specific analgesic effects by i.c.v. (C), i.t. (D), or i.pl. (E) of morphine in the EPW $(n=4)$. (F and G) Time course (F) and dose-dependency (G) of DAMGO-induced analgesia in the thermal test $(n=7$ to 8 ). The experimental paradigms were identical to the cases with morphine. (A) $* P<0.05$; **P $<0.01$, vs. Control (Cont) aCSF at each time point, two-way ANOVA followed by Tukey's multiple comparisons test (interaction: $\mathrm{F}_{18,196}=9.043, P<0.0001$; time: $\mathrm{F}_{6,196}=11.89$, $P<0.0001$; treatment: $\left.\mathrm{F}_{3,196}=527.5, P<0.0001\right)$. (B) ${ }^{*} P<0.05$, vs. Cont at dose 0 (means aCSF injection), one-way ANOVA followed by Dunnett's multiple comparisons test $\left(\mathrm{F}_{32} 28=47.38, P<0.0001\right.$ ).; ${ }^{\# \#} P<0.01$, vs. Cont at 0 (means aCSF injection), one-way ANOVA followed by Tukey's multiple comparisons test $\left(\mathrm{F}_{7,56}=158.3, P<0.0001\right)$. (C-E) $* P<0.05 ; * * P<0.01$, vs. time 0 at each column, one-way ANOVA followed by Tukey's multiple comparisons test. $\mathrm{F}_{11,36}=144.8, P<0.0001$ (C); $\mathrm{F}_{3,12}=17.88, P=0.0001$ (D); $\mathrm{F}_{11,36}=88.11, P<0.0001$ (E). $(\mathrm{F}) * P<0.05 ; * * P<0.01$, vs. Cont aCSF at each time point, two-way ANOVA followed by Tukey's multiple comparisons test (interaction: $\mathrm{F}_{18,189}=4.172, P<0.0001$, time: $\mathrm{F}_{6,189}=5.294$, $P<0.0001$, treatment: $\mathrm{F}_{3,189}=204.9, P<0.0001$ ). (G) $* P<0.05, * * P<0.01$, vs. Cont at dose 0 (means aCSF injection), one-way ANOVA followed by Dunnett's multiple comparisons test $\left(\mathrm{F}_{3,28}=15.86, P<0.0001\right.$ ).; ${ }^{\# \#} P<0.01$, vs. Cont at dose 0 (means aCSF injection), one-way ANOVA followed by Tukey's multiple comparisons test $\left(\mathrm{F}_{7,55}=48.81, P<0.0001\right)$. The number in each column represents the number of mice used. 
(Eco Realtime PCR System, Illumina, San Diego, CA) were as follows; for glyceraldehyde-3-phosphate dehydrogenase, 5'-TGCACCACCAA CTGCTTAGC-3' (forward) and 5'-GGCATGGACTGTGGTCATGAG$3^{\prime}$ (reverse); for NR2A, 5'-CCCCCTTCGTCATCGTAGAA-3' (forward) and 5' ${ }^{\prime}$-CAGAACCCCTTGCAGCACTT-3' (reverse); for NR2B, $5^{\prime}$-AAA GATGCCCACGAGAAA-3' (forward) and 5'-AGATGCGGGTGATTA TGCTC-3' (reverse); for NR1，5'-GTCCTCTGCCATGTGGTTTT-3' (forward) and 5'-GGTCATTGATGCCTGTGATG-3' (reverse); for PSD95, 5' -GACGCCAGCGACGAAGAG-3' (forward) and 5'-CTCGA CCCGCCGTTTG-3' (reverse); for BDNF, $5^{\prime}$ - CATAAGGACGCGGAC TTGTACA-3' (forward) and 5' - TGCGGCATCCAGGTAATTTT-3' (reverse); for MOPr, 5'-TCCTGGTCATGTATGTGATTGTAAGA-3' (forward) and 5'-CGTGCTAGTGGCTAAGGCATCT-3' (reverse). RPH3A, 5' -CCAAGACAACAGCAACCTGC-3' (forward) and 5'-CAT TCCACACAGGGTTCCGA-3' (reverse). The obtained data were normalized to the expression of glyceraldehyde-3-phosphate dehydrogenase and analyzed using the $\Delta \Delta \mathrm{Ct}$ Method.

Statistics. All data are expressed as the mean \pm S.E.M. Data were analyzed with Graphpad Prism 7.0 software (Graphpad Software, San Diego, CA) using the unpaired $t$ test, one-way ANOVA with Tukey's multiple comparisons test, or Dunnett's multiple comparisons test, and two-way ANOVA with Tukey's multiple comparisons test or Bonferroni's multiple comparisons test. Differences with a $P$ value of less than 0.05 were considered statistically significant.

\section{Results}

Lack of Morphine Analgesia in an Intermittent Cold Stress-Induced Widespread Pain Model. The ICS caused significant mechanical hyperalgesia at P8 after ICS exposure as shown in Fig. 1A. The threshold measured by Electronic von Frey Anesthesiometer with Rigid Tips was 5.83 $\pm 0.18 \mathrm{~g}(n=8)$ in ICS mice, whereas the threshold in control mice without stress was $9.65 \pm 0.19 \mathrm{~g}(n=8)$. When morphine at $0.3 \mathrm{nmol}$ was administered i.c.v., significant analgesia was observed at 10,20, and 30 minutes, and it was dose-dependent in the range between 0.03 and $0.3 \mathrm{nmol}$ at 20 minutes in control mice, whereas no morphine analgesia was observed in ICS mice (Fig. 1, A and B). In the EPW test using control mice, morphine (i.c.v.) significantly increased the nociceptive thresholds by electrical stimuli to the paw at 5,250 , and $2000 \mathrm{~Hz}$, as shown in Fig. 1C. When mice were treated with ICS, the thresholds at 250 and $2000 \mathrm{~Hz}$ were significantly decreased by approximately $45 \%$ and $20 \%$, whereas there was no change at $5 \mathrm{~Hz}$ compared with those with control mice. The i.c.v. treatment with morphine at $0.3 \mathrm{nmol}$ had no effect on these thresholds in ICS mice, whereas the i.t. and i.pl. treatments significantly increased the threshold only at $5 \mathrm{~Hz}$, but not at 250 or $2000 \mathrm{~Hz}$, as shown in Fig. 1, D and E, respectively. The dose selection for morphine treatments through different administration routes followed the previous report (Rashid et al., 2004). The lack of MOPr agonist-induced analgesia in ICS mice was also observed with the peptide agonist DAMGO at $0.3,1$, and 3 pmol (i.c.v.) in the thermal nociception test, as shown in Fig. 1, F and G.

Lack of Influence of MOPr Deficiency on ICS-Induced Pain. The thermal and mechanical hyperalgesia in ICS mice was not affected in $\mathrm{MOPr}^{-1}$ mice (Fig. 2, A and B). Similar results were also observed in the hyperalgesia in mechanical muscle pain (Fig. 2C) and EPW (250 and $2000 \mathrm{~Hz}$ ) tests (Fig. 2, $\mathrm{D}$ and $\mathrm{E})$, whereas no change was observed in the EPW $(5 \mathrm{~Hz})$ test (Fig. 2F). In addition, there were no statistical differences between wild-type (WT) control and $\mathrm{MOPr}^{-1-}$ mice (Fig. 2).

Lack of Morphine Analgesia Remained in ICS Mice that Lost the Hyperalgesia. In $\mathrm{LPA}_{1}^{-1}$ mice without ICS, there was no difference in the basal pain threshold and brain morphine analgesia compared with WT mice (Fig. 3A). In contrast, in ICS-treated $\mathrm{LPA}_{1}^{-1}$ mice, the basal pain threshold was elevated to the naïve level seen in WT mice not subjected to ICS exposure, but brain morphine analgesia was still lost (Fig. 3B). The lack of morphine analgesia remained
A

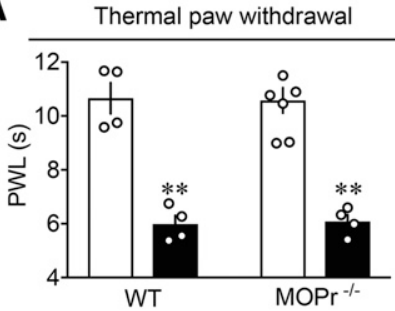

D

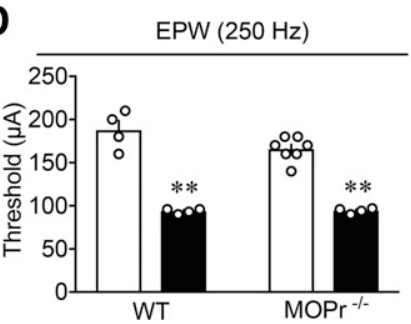

B

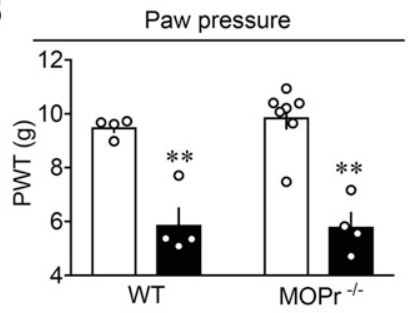

E

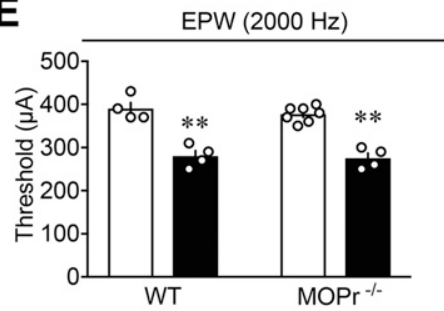

C

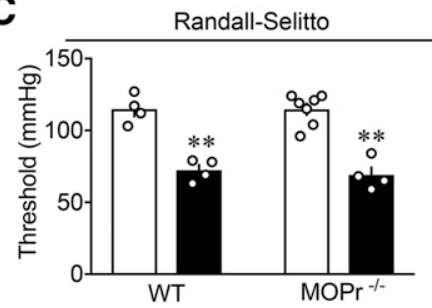

$\mathbf{F}$

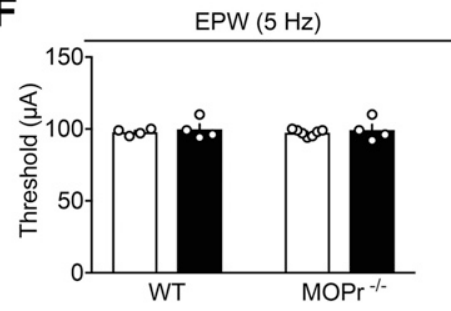

Fig. 2. Lack of influence by MOPr gene-deficiency on ICS-induced hyperalgesia. Results represent the thresholds of WT and MOPr gene-deficient $\left(\mathrm{MOPr}^{-1}\right)$ mice in the absence (Cont) or presence of ICS at P5 in thermal paw withdrawal test (A, in second/s), paw pressure test (B, in gram/g), RandallSelitto test (C, in $\mathrm{mmHg})$ and EPW test [(D) at $250 \mathrm{~Hz}$, (E) at $2000 \mathrm{~Hz},(\mathrm{~F})$ at $5 \mathrm{~Hz}$, all in microampere] $(n=4)$.; ** $P<0.01$, vs. Cont, two-way ANOVA followed by Bonferroni's multiple comparisons test. Interaction: $\mathrm{F}_{1,15}=0.0339, P=0.8574$, mouse: $\mathrm{F}_{1,15}=9.25 \mathrm{e}-005, P=0.9925$, treatment: $\mathrm{F}_{1,15}=$ 92.66, $P<0.0001$ (A), interaction: $\mathrm{F}_{1,15}=0.2135, P=0.6506$, mouse: $\mathrm{F}_{1,15}=0.1015, P=0.7544$, treatment: $\mathrm{F}_{1,15}=63.53, P<0.0001$ (B), interaction: $\mathrm{F}_{1,15}=0.1147, P=0.7395$, mouse: $\mathrm{F}_{1,15}=0.1199, P=0.7340$, treatment: $\mathrm{F}_{1,15}=88.41, P<0.0001(\mathrm{C})$, interaction: $\mathrm{F}_{1,15}=3.09, P=0.0991$, mouse: $\mathrm{F}_{1}$, $15=2.819, P=0.1138$, treatment: $\mathrm{F}_{1,15}=169.8, P<0.0001$ (D), interaction: $\mathrm{F}_{1,15}=0.129, P=0.7245$, mouse: $\mathrm{F}_{1,15}=0.6661, P=0.4272$, treatment: $\mathrm{F}_{1}$, $15=94.01, P<0.0001(\mathrm{E})$, interaction: $\mathrm{F}_{1,15}=0.001425, P=0.9704$, mouse: $\mathrm{F}_{1,15}=0.03015, P=0.8645$, treatment: $\mathrm{F}_{1,15}=0.6526, P<0.4318(\mathrm{~F})$. 
Thermal paw withdrawal test
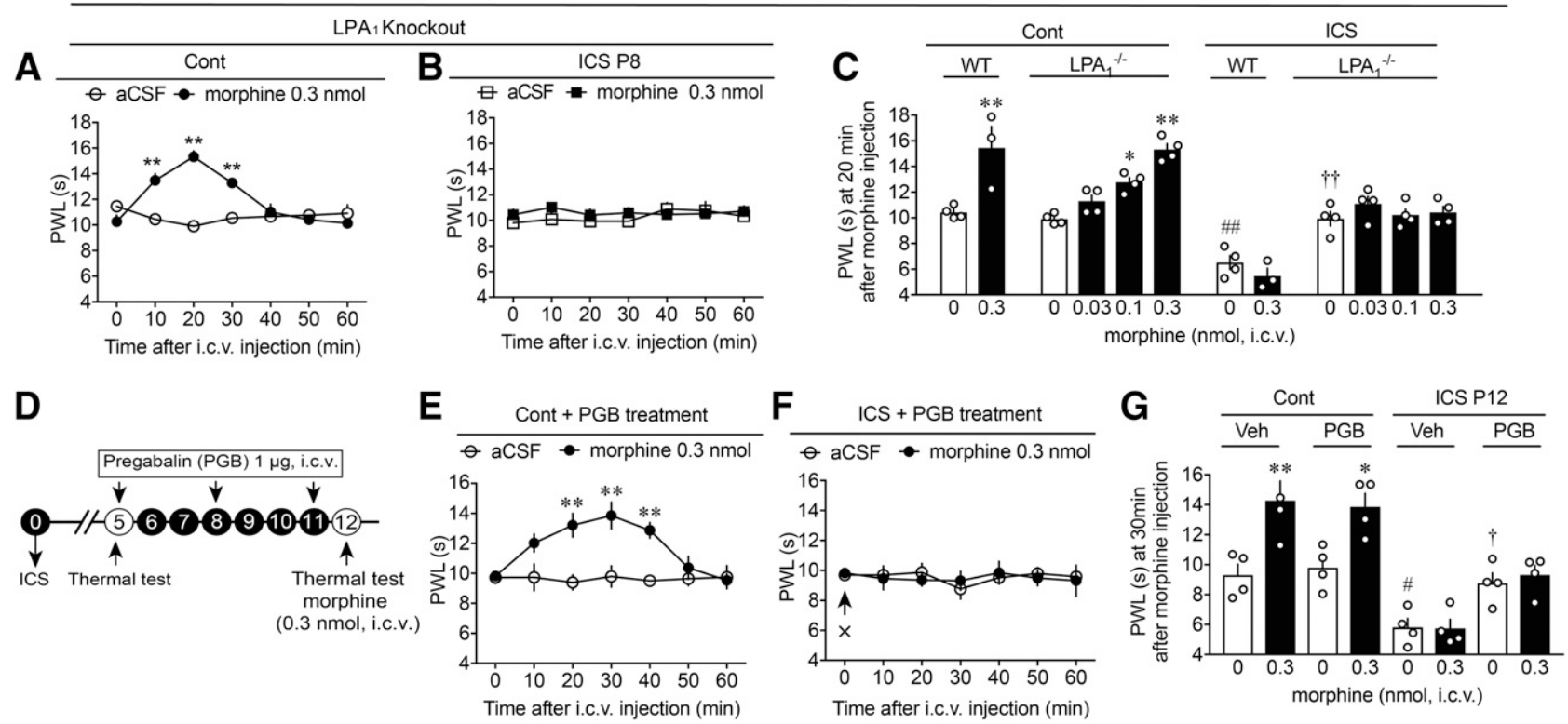

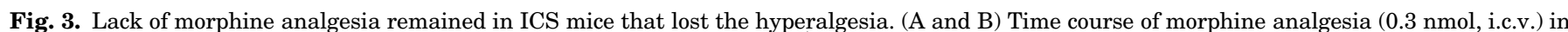

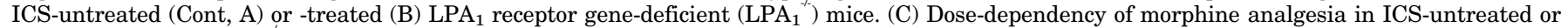

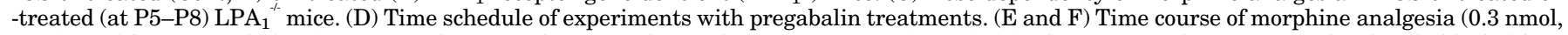

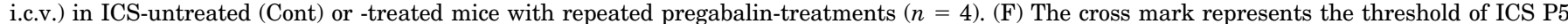

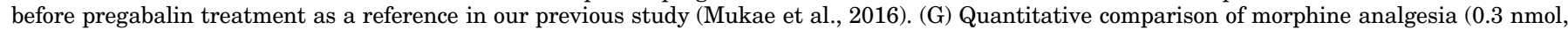

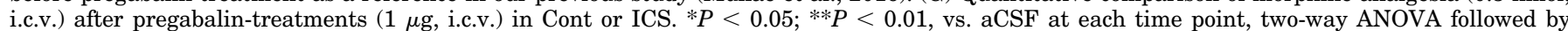

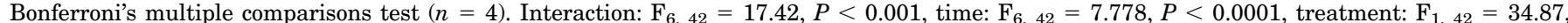

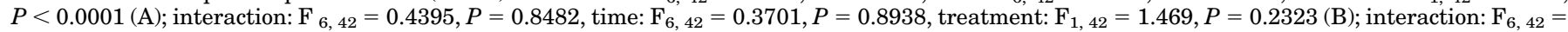

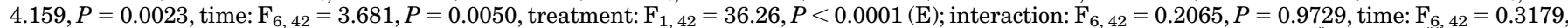

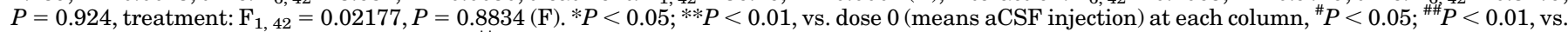

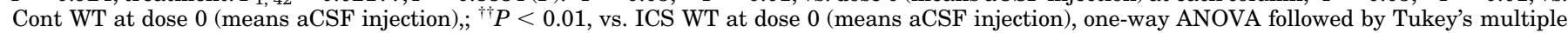

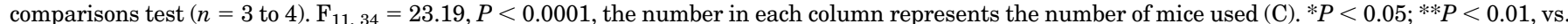

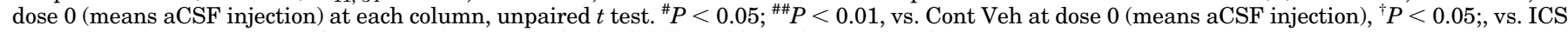
$\mathrm{P} 12$ Veh at dose 0 (means aCSF injection), one-way ANOVA followed by Tukey's multiple comparisons test $(n=4)$. $\mathrm{F}_{3}, 12=6.91, P=0.0059(\mathrm{G})$.

unchanged in $\mathrm{LPA}_{1}^{-/}$mice, which completely lost the hyperalgesia (Fig. 3C). Similar results were also observed in mice repeatedly treated with pregabalin (i.c.v.), which also completely lost the hyperalgesia (Fig. 3, D-F). In pregabalintreated mice without ICS, there was no difference in the basal pain threshold and brain morphine analgesia (Fig. 3E). Although the basal pain threshold was elevated to the naïve level in ICS- and pregabalin-treated mice, the lack of brain morphine analgesia remained unchanged (Fig. 3F).

Involvement of the NR2A Subunit in the Lack of Brain Morphine Analgesia in ICS Mice. Treatment with morphine at $0.3 \mathrm{nmol}$ (i.c.v.) showed significant thermal analgesic effects in control mice but not in ICS mice (Fig. 4, A and $\mathrm{B})$. When NR2A gene-deficient (NR2A ${ }^{-1}$ ) mice were used, the lack of brain morphine analgesia at different doses (0.03-0.3 nmol, i.c.v.) was completely reversed, though there was no significant change in thermal hyperalgesia (Fig. 4, C-E). Significant recovery of morphine analgesia was also observed when $0.03 \mathrm{nmol}$ (i.c.v.) of (R)-CPP, an NR2A antagonist, but not 1 nmol (i.c.v.) Ro 04-5595, an NR2B antagonist was given 20 minutes before morphine administration (Fig. 4F), though (R)-CPP and Ro 04-5595 alone have no effect on the thermal nociceptive threshold (Supplemental Fig. 1, A-D).

Recovery of Morphine Analgesia by Microinjection of siRNA NR2A into the PAG and Dorsal Raphe. The reversal of the lack of morphine analgesia was also observed following microinjection of siRNA NR2A, which was performed with a subsequent thermal test at P5 (Fig. 5A). Figure 5B shows the loci where siRNA NR2A was microinjected into PAG regions including the VLPAG and the ventrolateral, dorsal, and ventral parts of the dorsal raphe (DRVL, DRD, and DRV, respectively). As shown in Fig. 5C, mice given siRNA NR2A showed significant morphine analgesia at P5, but those given small interfering control RNA treatment did not. However, the microinjection of siRNA NR2A into regions ventral to the DR, such as the superior cerebellar peduncle, ventral tegmental nucleus and subpedencular tegmental nucleus did not show significant morphine analgesia (Supplemental Fig. 2). In a separate experiment, siRNA NR2A microinjection into the PAG region $(213 \pm 20 \mu \mathrm{g}$ protein) significantly decreased the gene expression of NR2A at P5 (Fig. 5D). When average morphine analgesia was estimated by subtraction of the threshold before morphine from that at 10 minutes after morphine, it was calculated to be 3.4 and 3.6 seconds in control mice with and without siRNA, respectively, whereas it was 2.9 and 0.3 seconds in ICS mice with and without siRNA, respectively (Fig. 5E). However, no significant morphine analgesia in ICS mice was observed after siRNA microinjection into the VTA (Fig. 5E).

To examine the possible role of another antiopioid vasopressin system, SSR, a vasopressin $1 \mathrm{~b}$ receptor antagonist (1 nmol, i.c.v.) was given 10 minutes prior to morphine treatment in ICS mice. However, the lack of morphine analgesia was not reversed by this antagonist (Fig. $5 \mathrm{~F}$ ), which alone has no effect on the nociceptive threshold in control and ICS mice (Supplemental Fig. 1, E and F). When quantitative reverse transcription polymerase chain reaction analysis was performed using PAG 
Thermal paw withdrawal test
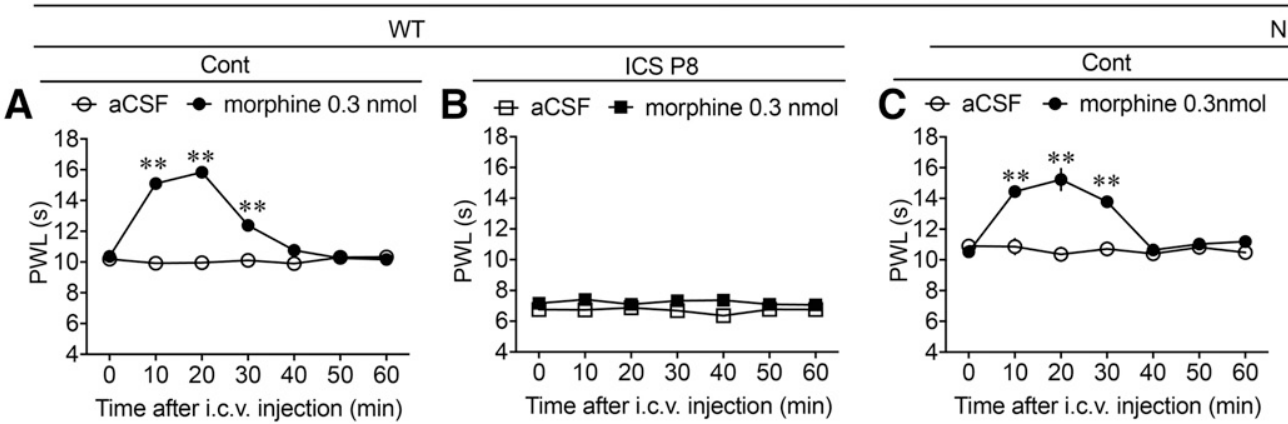

$\mathrm{RR}^{-1-}$

Time after i.c.v. injection ( $\mathrm{min}$ )
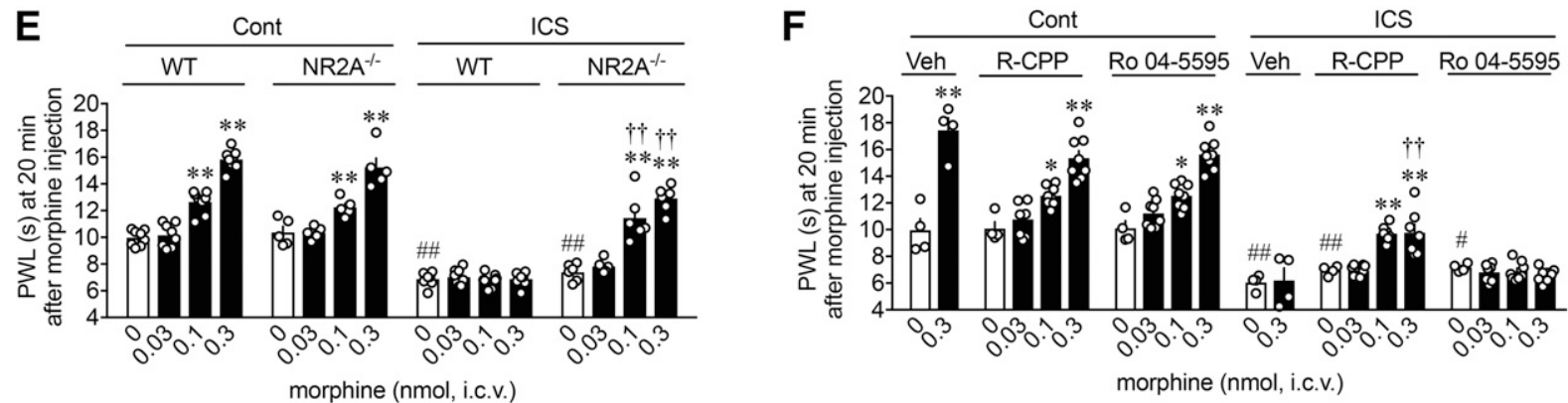

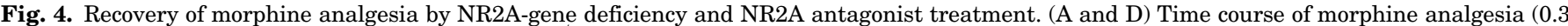

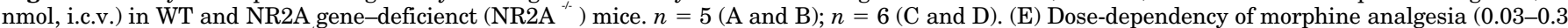

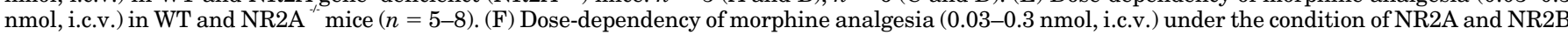

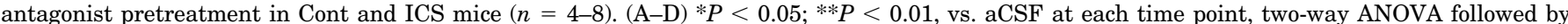

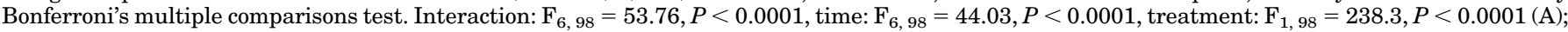

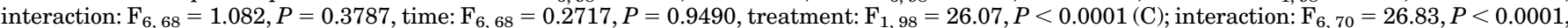

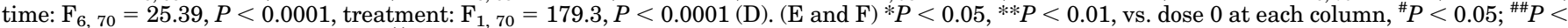

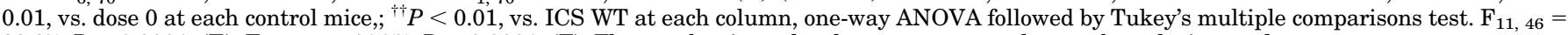
32.37, $P<0.0001$; (E), $\mathrm{F}_{19,107}=61.77, P<0.0001$; $(\mathrm{F})$. The number in each column represents the number of mice used

regions, however, we failed to detect any significant difference in the gene expressions of NMDA receptor subunits, PSD95, BDNF, MOPr, and RPH3A in the PAG between control and ICS mice at P5 (Supplemental Fig. 3, A-H).

Beneficial Effects of Clinically Available Medicines Possessing NMDA Receptor Antagonist Activity. The systemic administration of dextromethorphan (DXM) at $3 \mathrm{mg} / \mathrm{kg}$ (i.p.) showed significant antihyperalgesia (or analgesia) in ICS mice with a peak effect at 30-60 minutes (Fig. 6A). However, the beneficial action was only observed at $3 \mathrm{mg} / \mathrm{kg}$ of DXM (i.p.) but not at lower doses (Fig. 6B). When DXM was used as a pretreatment (30 minutes) at $1 \mathrm{mg} / \mathrm{kg}$ (i.p.) (a subeffective dose), the dosedependent morphine analgesia was recovered in ICS mice, whereas no significant change was observed in control mice (Fig. 6C).

As shown in Fig. 6D, i.c.v. injection of methadone, which has both MOPr agonist and NMDA receptor antagonist activities, showed significant analgesic effects in control mice with a peak effect at 20 minutes. Equipotent analgesic effects of methadone were also observed in ICS mice (Fig. 6E) but were abolished in $\mathrm{MOPr}^{{ }^{+-}}$mice (Fig. 6, F and G). Figure 6H showed that equipotent methadone analgesia was observed both in control and ICS mice when different doses (0.03-3 nmol, i.c.v.) were used. Methadone also showed similar time course and dose-related analgesic actions when given through a systemic route (s.c.), as shown in Fig. 6, I and J.

\section{Discussion}

We have previously developed chronic widespread pain models using ICS or IPS, which have similar features to those clinically seen in patients with fibromyalgia (Clauw, 2014) in terms of pathophysiology and pharmacotherapy (Nishiyori and Ueda, 2008; Nishiyori et al., 2010, 2011; Ueda and Neyama, 2017). Both models manifest unique femalepredominant hyperalgesia particularly after gonadectomy is performed, though no significant difference in the degree of hyperalgesia between male and female mice was observed in both ICS and IPS models without gonadectomy (Nishiyori and Ueda, 2008; Ueda and Neyama, 2017). Because it has been shown that NMDA receptor antagonism disrupts the development of morphine analgesic tolerance in male but not female mice (Bryant et al., 2006), we used male mice to study opioid involvement in the abnormal pain and lack of morphine analgesia.

It is widely known that pain inhibition by exogenous morphine or endogenous opioids is mediated through the activation of bulbospinal monoaminergic systems (Sato and Takagi, 1971; Deakin and Dostrovsky, 1978; Takagi et al., 1978; Fields et al., 1983; Basbaum and Fields, 1984; Millan, 2002). These observations may be related to the mechanisms underlying chronic pain and lack of morphine analgesia in ICS-model mice, which show an inhibition of brain morphine-induced elevation of the 5-HT turnover rate in the spinal cord (Nishiyori et al., 2010). Further, basal norepinephrine turnover rate in the spinal cord is decreased in IPS-model mice (Ueda and Neyama, 2017). There are interesting reports that show acute stress causes analgesia by the actions of endogenous opioids (Ferdousi and Finn, 2018), whereas heavy stress causes hyperalgesia (Jennings et al., 2014). Although no 


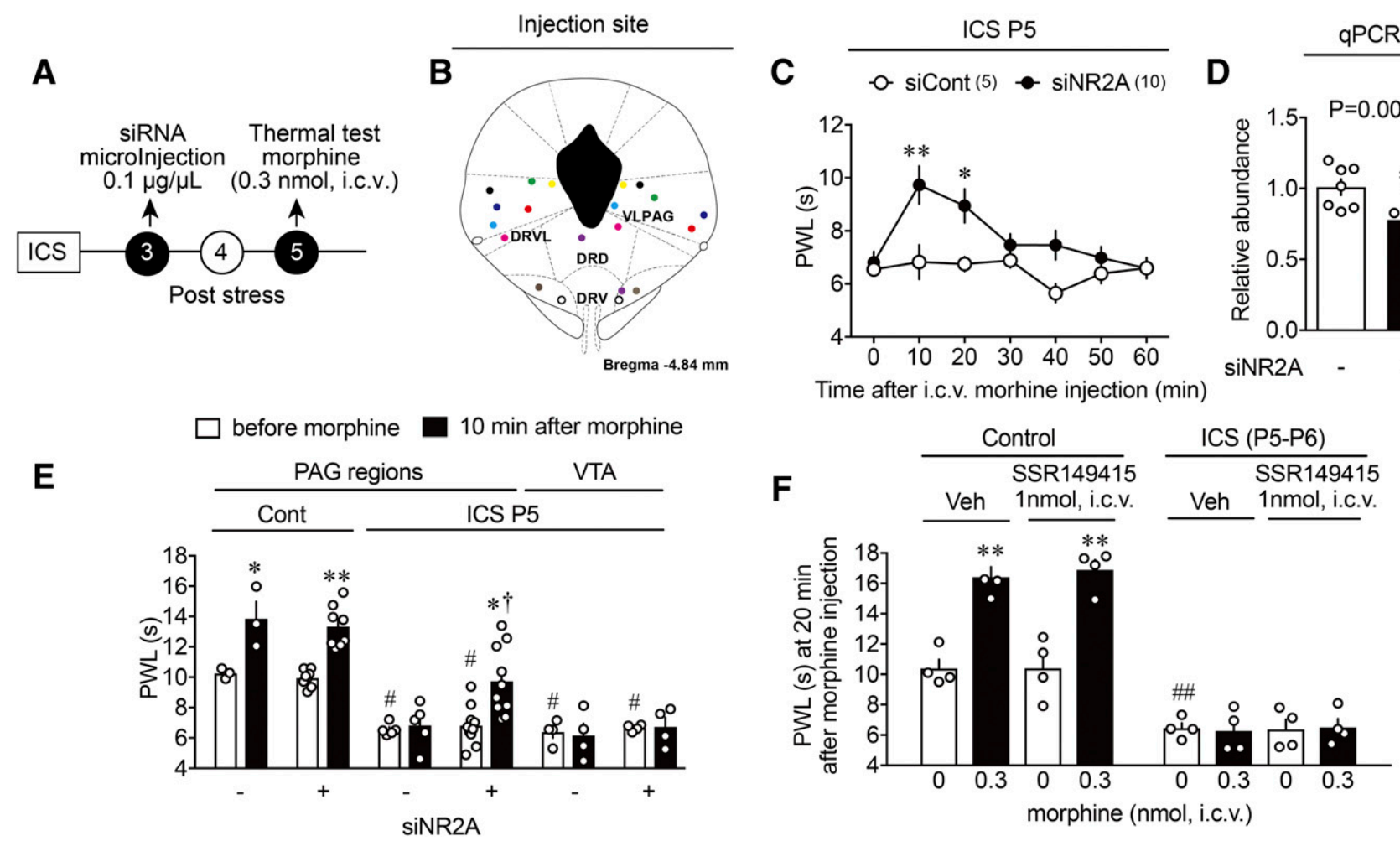

Fig. 5. Selective recovery of morphine analgesia by microinjection of siRNA NR2A into PAG of ICS-treated mice. (A) Time schedule of experiment. (B) Confirmed sites of siRNA microinjection in PAG area. (C) Morphine analgesia ( $0.3 \mathrm{nmol}$, i.c.v.) 2 days after microinjection of siRNA NR2A into PAG in the thermal paw withdrawal test $(n=5-10)$. (D) Decreased NR2A gene expression 2 days after siRNA NR2A microinjection into PAG $(n=4)$. (E) Morphine analgesia $(0.3 \mathrm{nmol}$, i.c.v) after siNR2A microinjection into PAG or VTA. (F) Morphine analgesia (0.3 nmol, i.c.v.) 10 minutes after SSR149415 treatment $(1 \mathrm{nmol}$, i.c.v.). (C) $* P<0.05 ; * * P<0.01$, vs. siCont at each time point, two-way ANOVA followed by Bonferroni's multiple comparisons test. Interaction: $\mathrm{F}_{6,90}=2.006, P=0.0729$, time: $\mathrm{F}_{6,90}=3.0085, P<0.0085$, treatment: $\mathrm{F}_{1,90}=16.17, P<0.0001$. The number of parenthesis represents the number of mice used. (D) $* P<0.05 ; * * P<0.01$, vs. siNR2A (-), unpaired $t$ test. (E) $* P<0.05 ; * * P<0.01$, vs. before morphine at each column, unpaired $t$ test, ${ }^{\#} P<0.05$; ${ }^{\# \#} P<0.01$, vs. Cont before morphine at each column, ${ }^{\dagger} P<0.05 ;{ }^{\dagger \dagger} P<0.01$, vs. PAG region ICS P5 siNR2A (-) 10 minute after morphine, one-way ANOVA followed by Tukey's multiple comparisons test $\left(\mathrm{F}_{11,56}=20.89, P<0.0001\right)$. The number in each column represents the number of mice used. In (F), ${ }^{*} P<0.05$; ${ }^{* *} P<0.01$, vs. dose 0 (means aCSF injection) at each column, ${ }^{\#} P<0.05 ;{ }^{\# \#} P<0.01$, vs. Cont Vehicle (Veh) at dose 0 (means aCSF injection), one-way ANOVA followed by Tukey's multiple comparisons test $\left(\mathrm{F}_{7,24}=45.19, P<0.0001\right)$. siCont, small interfering control.

experimental evidence is available to explain the mechanisms underlying this hyperalgesia, it is interesting to speculate that it may be due in part to the analgesic tolerance caused by a repeated stress-induced release of endogenous opioids. Related reports have shown that the opioid binding seen in a Positron emmision tomography (PET) study and opioid action-related functional activity seen in an Functional Magnetic Resonance Imaging (fMRI) study are negatively correlated to degree of pain in patients with fibromyalgia (Harris et al., 2007; Schrepf et al., 2016). However, in the present study, MOPr ${ }^{-1}$ mice did not show attenuation of ICSinduced hyperalgesia, suggesting that endogenous opioid mechanisms are unlikely to be involved in abnormal pain mechanisms.

The lack of morphine analgesia in ICS mice, in contrast, is consistent with a clinical study that showed that there is no conclusive evidence that opioids are effective in the treatment of fibromyalgia (Goldenberg et al., 2016). The next question is whether hyperalgesia status reduces the potency of morphine analgesia. We previously reported that type 1 lysophosphatidic acid receptor signaling plays key roles in the development and maintenance of several widespread fibromyalgia-like pain models (Ueda and Neyama, 2017), as well as many other chronic pain models, such as peripheral nerve injury (Inoue et al., 2004), paclitaxel (Uchida et al., 2014)-induced peripheral neuropathic pain and post stroke-type central neuropathic pain (Ueda et al., 2018b) models. However, in the present study the lack of morphine analgesia remained unchanged even when the ICS-induced pain was abolished in $\mathrm{LPA}_{1}{ }^{-1}$ mice. Because the lack of morphine analgesia also remained unchanged in mice treated with repeated i.c.v. treatments with pregabalin, which abolished the hyperalgesia, it is evident that lack of morphine analgesia and hyperalgesia in the ICS model have independent mechanisms.

Our next concerns are what mechanisms underlie the lack of morphine analgesia in the ICS model. Our initial speculation is based on the findings that stress-induced analgesia is blocked by opioid antagonists and is partly mediated by endogenous opioids (e.g., $\beta$-endorphin) (Butler and Finn, 2009; Ferdousi and Finn, 2018). Intense and repeated stress may cause excess and continuous release of endogenous opioids, which in turn lead to a downregulation of opioid binding, as seen in clinical findings using PET analysis (Harris et al., 2007). The continuous stimulation of MOPr by endogenous opioids may also cause analgesic tolerance, possibly due to a synaptic plasticity caused by antiopioid mechanisms. Previously, we have reported that repeated morphine administration causes analgesic tolerance, which was abolished in NR2A ${ }^{-}$mice (Inoue et al., 2003). In this study, the morphine analgesic tolerance was recovered by 


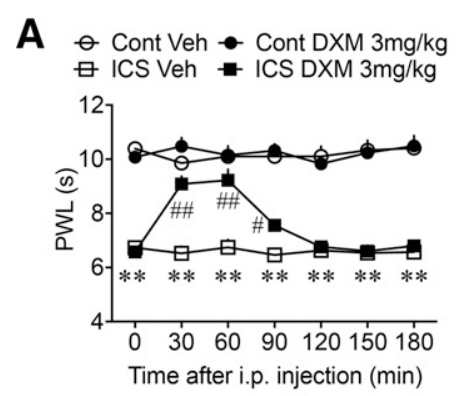

B

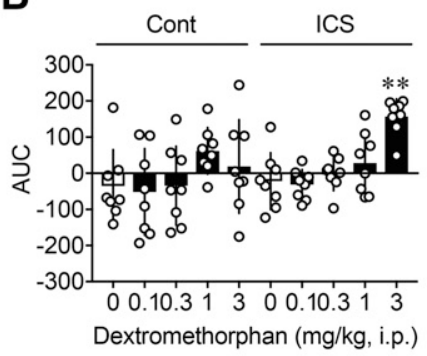

C

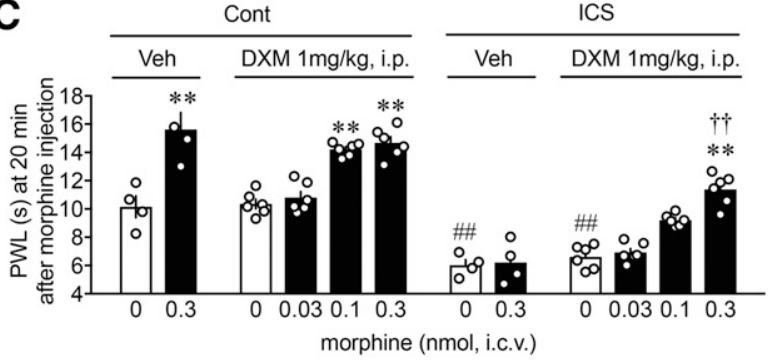

WT
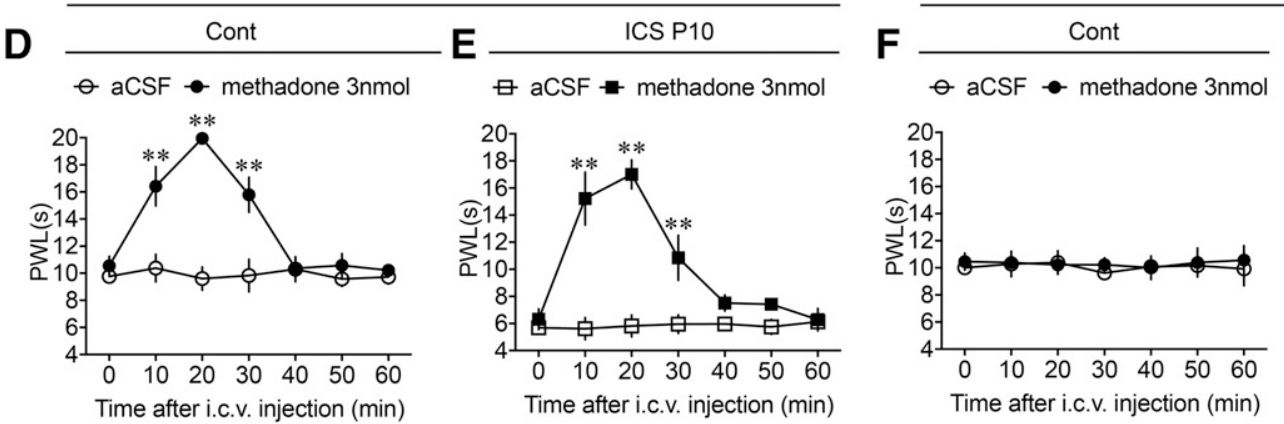

$\mathrm{MOPr}^{-/}$
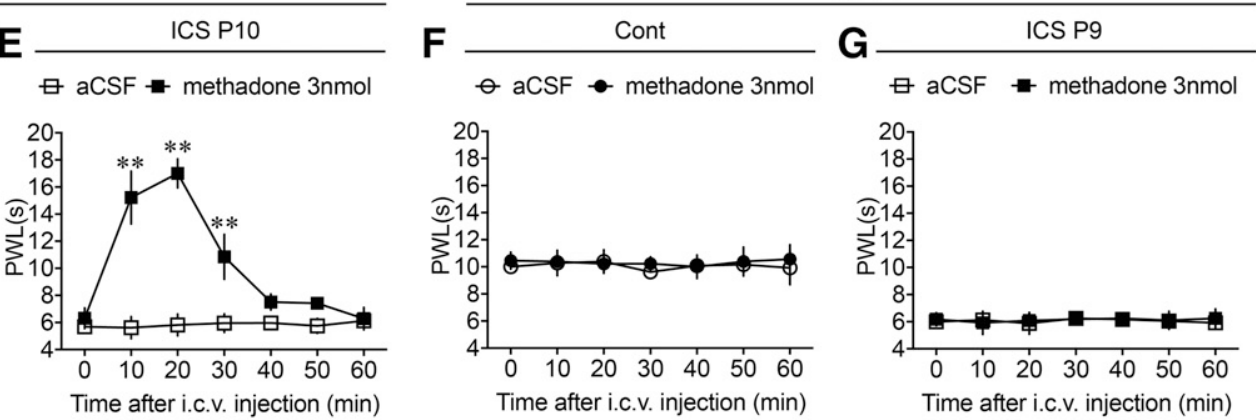

H
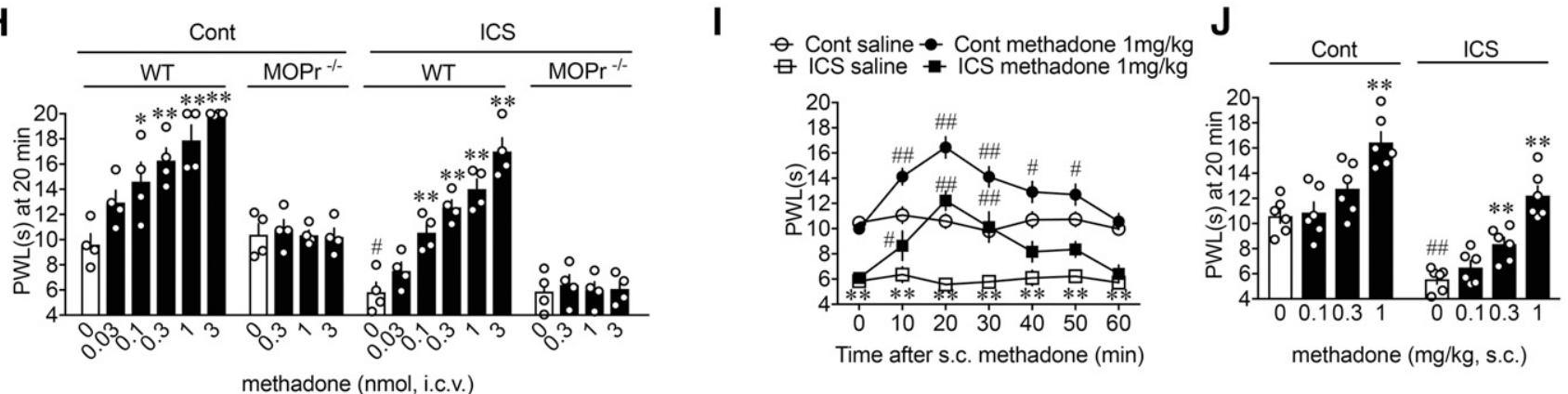

Fig. 6. Beneficial effects of dextromethorphan and methadone to treat ICS-induced pain. All experiments were performed in the thermal paw withdrawal test. (A) Time course of DXM effects on the nociceptive responses in Cont or ICS mice $(n=8)$. (B) Dose-dependency of DXM-induced analgesia. Results were represented by AUC, in which analgesia was evaluated by the threshold at each time point $(0,30,60,90,120,150,180$ minutes $)$ - threshold at time 0 , followed by summation of thresholds at seven time points and multiplication by 180. (C) Dose-dependency of morphine analgesia (i.c.v.) after the pretreatment with DXM $(1 \mathrm{mg} / \mathrm{kg}$, i.p.) in Cont or ICS mice $(n=4-6)$. (D-G) Time course of methadone (3 nmol, i.c.v.) analgesia in WT Cont (D), WT ICS (E), MOPr ${ }^{-1}$ Cont (F), or MOPr ${ }^{-1 C S ~}(\mathrm{G})(n=4)$. (H) Dose-dependency of methadone analgesia (i.c.v.) in WT or MOPr ${ }^{-1-}(n=4)$. (I and J) Time course (I) and dose-dependency (J) of methadone analgesia at $1 \mathrm{mg} / \mathrm{kg}$ s.c. $(n=6) .{ }^{*} P<0.05 ; * * P<0.01$, vs. Cont Veh, ${ }^{\#} P<0.05 ;{ }^{* \#} P<0.01$, vs. ICS Veh, two-way ANOVA followed by Tukey's multiple comparisons test. Interaction: $\mathrm{F}_{18,196}=5.18, P<0.0001$, time: $\mathrm{F}_{6,196}=4.296, P=0.0004$, treatment: $\mathrm{F}_{3,196}=326.2, P<0.0001$ (A). ${ }^{*} P<0.05$; $* * P<0.01$, vs. ICS at dose 0 (means Veh injection), one-way ANOVA followed by Dunnett's multiple comparisons test. $\mathrm{F}_{4,35}=11.8, P<0.0001$ (B). ${ }^{*} P<0.05$; ${ }^{*} P<0.01$, vs. dose 0 (means aCSF injection) at each column, ${ }^{\#} P<0.05$; ${ }^{\# \#} P<0.01$, vs. Cont Veh, ${ }^{\dagger} P<0.05 ;{ }^{\dagger \dagger} P<0.01$, vs. ICS Veh $(0.3 \mathrm{nmol})$, one-way ANOVA followed by Tukey's multiple comparisons test. $\mathrm{F}_{11,52}=48.78, P<0.0001$, the number in each column represents the number of mice used (C). (D-G) $* P<0.05$; ** $P<0.01$, vs. aCSF at each time point, two-way ANOVA followed by Bonferroni's multiple comparisons test. Interaction: $\mathrm{F}_{6,42}=10.47, P<0.0001$, time: $\mathrm{F}_{6,42}=10.47, P<0.0001$, treatment: $\mathrm{F}_{1,42}=58.33, P<0.0001$ (D); interaction: $\mathrm{F}_{6,42}=11.89, P<0.0001$, time: $\mathrm{F}_{6,42}=11.21, P<0.0001$, treatment: $\mathrm{F}_{1,42}=74.14, P<0.0001(\mathrm{E})$; interaction: $\mathrm{F}_{6,42}=0.08182, P=0.9977$, time: $\mathrm{F}_{6,42}=0.07274, P=0.9983$, treatment: $\mathrm{F}_{1,42}=0.3618, P=0.5508(\mathrm{~F})$; interaction: $\mathrm{F}_{6,42}=0.05405, P=0.9993$, time: $\mathrm{F}_{6,42}=0.04904, P=0.9995$, treatment: $\mathrm{F}_{1,42}=0.08046, P=0.7781(\mathrm{G}) * P<0.05, * * P<0.01$, vs. dose 0 (means aCSF injection) at each column, one-way ANOVA followed by Dunnett's multiple comparisons test (Cont: $\mathrm{F}_{5,18}=12.4, P<0.0001$, ICS: $\mathrm{F}_{5,18}=27.17, P<0.0001$ ). ${ }^{\#} P<0.05$; ${ }^{\# \#} P<0.01$, vs. Cont at dose 0 (means aCSF injection) at each column, one-way ANOVA followed by Tukey's multiple comparisons test $\mathrm{F}_{3,12}=8.421, P=0.0028(\mathrm{H}) . * P<0.05 ; * * P<0.01$, vs. Cont Saline at each time point, two-way ANOVA followed by Tukey's multiple comparisons test (interaction: $\mathrm{F}_{18,140}=3.721, P<0.0001$, time: $\mathrm{F}_{6,140}=$ 10.95, $P<0.0001$, treatment: $\mathrm{F}_{3,140}=142.5, P<0.0001$ ). ${ }^{\#} P<0.05$; ${ }^{\# \#} P<0.01$, vs. time 0 (means before methadone injection), two-way ANOVA followed by Dunnett's multiple comparisons test. Interaction: $\mathrm{F}_{18,140}=3.721, P<0.0001$, time: $\mathrm{F}_{6,140}=10.95, P<0.0001$, treatment: $\mathrm{F}_{3,140}=142.5$, $P<0.0001$ (I). ${ }^{*} P<0.05,{ }^{* *} P<0.01$, vs. dose 0 (means aCFS injection) at each column, one-way ANOVA followed by Dunnett's multiple comparisons test. Cont: $\mathrm{F}_{3,20}=11.81, P=0.0001$, ICS: $\mathrm{F}_{3,20}=26.94, P<0.0001$; ${ }^{\#} P<0.05$; ${ }^{\# \#} P<0.01$, vs. Cont at time 0 , unpaired $t$ test.

NR2A gene rescue into the PAG or VTA of NR2A ${ }^{-1}$ mice. It should be noted that the lack of brain morphine analgesia in the ICS model was also completely recovered in NR2A ${ }^{-1}$ mice. This finding was further supported by the findings that morphine analgesia was recovered by i.c.v. treatment with (R)-CPP, an NR2A antagonist (Feng et al., 2005), but not with Ro 04-5595, an NR2B antagonist (Mutel et al., 1998). More specifically, the microinjection of siRNA NR2A into PAG areas including the DR significantly reversed the lack of morphine analgesia. Because no significant recovery of morphine analgesia was observed by microinjection of siRNA NR2A into the VTA, the antiopioid involvement in the ICS model seems to be slightly different to the analgesic tolerance induced by repeated morphine treatments, where the NR2A gene rescue 
into the VTA of NR2A ${ }^{-1}$ mice reproduced morphine analgesic tolerance (Inoue et al., 2003). Thus, it appears that the NR2ANMDA receptor system may be one of the key mechanisms underlying the lack of morphine analgesia in the ICS model. However, the molecular mechanisms involved in the enhanced antiopioid NR2A-NMDA receptor system remain unclear. Although the protein expression of NR2A was upregulated in the PAG following repeated morphine treatments (Inoue et al., 2003), we failed in the present study to detect any significant gene expression of NMDA receptor subunits and closely related proteins, such as MOPr, PSD95 (Niethammer et al., 1996), BDNF (Matsushita et al., 2013), and RPH3A (Stanic et al., 2015). More detailed studies to evaluate the changes in the glutamate synthesis, release, and post-NMDA receptor synaptic plasticity remain elusive.

Most recently, vasopressin $1 \mathrm{~b}$ receptor has been reported to play roles in morphine analgesic tolerance as another type of antiopioid system (Koshimizu et al., 2018). However, we failed to detect the recovery of morphine analgesia by SSR injection (i.c.v.) at a dose 100-fold higher than that in the report (Koshimizu et al., 2018) in which repeated treatments with $0.01 \mathrm{nmol}$ (i.c.v.) of SSR prior to every treatment of morphine suppressed morphine analgesic tolerance. Details of the difference in lack of morphine analgesia in these conditions remain to be determined, but it may be hypothesized that high doses of morphine given exogenously may directly activate not only neurons but also glial cells, followed by production of unidentified molecules, which in turn affect the glutamate/ NMDA receptor systems (van Weering et al., 2011; Wang et al., 2017).

In the present study, we demonstrated clinically beneficial information through the use of existing medicines. DXM has been reported to possess NMDA receptor antagonist activity as well as activities in serotonin, norepinephrine transporters/ receptors, nicotinic receptors, potassium channels, and voltage-gated calcium channels (Taylor et al., 2016). Accordingly, the analgesic activity induced by high doses of DXM may be related to the blockade of spinal NMDA receptor signaling and unidentified targets, which are independent of brain antiopioid mechanisms. To avoid the analgesic potency by DXM alone in the present study, we used $1 \mathrm{mg} / \mathrm{kg}$ of DXM, which has no significant analgesic effects, and examined whether morphine analgesia is recovered by DXM. The present study successfully showed the recovery of equipotent dose-related morphine analgesic activities in ICS-treated mice. Therefore, the recovery of lost morphine analgesia is presumed to block the NMDA receptor-mediated counterbalance mechanisms in the brain.

In this context, methadone seems to be a more ideal treatment of ICS-induced hyperalgesia because this compound possesses both MOPr agonist and NMDA receptor antagonist activities (Ebert et al., 1995; Gorman et al., 1997). Our speculation was successfully evidenced by the present study, in which the central and systemic administration of methadone alone showed significant and potent analgesic actions in ICS mice and the analgesic actions of central methadone were completely blocked in $\mathrm{MOPr}^{-1}$ mice. Thus, methadone could be used to treat this type of stress-related widespread pain insensitive to morphine or related opioids, though the side effects may limit the clinical use of methadone (Webster, 2013). Further, the continuous use of pure opioid agonists at elevated doses in these patients is not recommended because this may be one of the causes the current opioid epidemic/crisis.

Finally, to support the hypothesis that excess release of endogenous opioids by repeated stress in the ICS model may cause endogenous opioid analgesic tolerance, future studies should examine whether the lack of MOPr agonist-induced analgesia is affected in $\beta$-endorphin or prepro-enkephalin gene deficient mice. In addition, the possibility that repeated stress directly facilitates the glutamate-NMDA receptor system, which works as a negative regulator of MOPr-mediated analgesia, should be also taken into consideration in further studies.

In conclusion, we demonstrated that the lack of morphine analgesia in ICS-induced fibromyalgia-model mice may be caused by the counterbalance through an endogenous antiopioid glutamate/NMDA receptor system. Specifically, the NR2A subunit in the PAG and DR, but not the VTA, plays key roles in this system, though the involvement of other brain loci remains to be determined. As the present study demonstrated that the ICS-induced pain was suppressed by methadone, which is reported to have significant side effects, the development of hybrid compounds with MOPr agonist and NMDA receptor antagonist activities, but less adverse effects would be promising for pain suppression in patients with FM.

\section{Acknowledgments}

The authors are grateful to Takehiro Mukae, Chiho Miyama, and Souichiro Kawamoto for the technical help of evaluation in the pain and to Jerold Chun, Brigitte Kieffer, Takaaki Koshimizu for kind gifts of $\mathrm{LPA}_{1}{ }^{-1}$ mice, $\mathrm{MOPr}$ mice, and SSR149415, respectively. The authors have no conflict of interest to declare. This work was supported in part by Grants-in-Aid for the Platform for Drug Discovery, Informatics, and Structural Life Science [16am0101012j0005] (H.U.) from the Japan Agency for Medical Research and Development, Japan; KAKENHI JP17H01586 (H.U.) and JP26253077 (H.U.) from JSPS.

\section{Authorship Contributions}

Participated in study design: Ueda.

Conducted experiments: Neyama, Dozono.

Performed data analysis: Neyama.

Wrote or contributed to the writing of the manuscript: Neyama, Ueda.

\section{References}

Basbaum AI and Fields HL (1984) Endogenous pain control systems: brainstem spinal pathways and endorphin circuitry. Annu Rev Neurosci 7:309-338.

Bryant CD, Eitan S, Sinchak K, Fanselow MS, and Evans CJ (2006) NMDA receptor antagonism disrupts the development of morphine analgesic tolerance in male, but not female C57BL/6J mice. Am J Physiol Regul Integr Comp Physiol 291: R315-R326.

Butler RK and Finn DP (2009) Stress-induced analgesia. Prog Neurobiol 88:184-202. Clauw DJ (2014) Fibromyalgia: a clinical review. JAMA 311:1547-1555.

Cooper TE, Chen J, Wiffen PJ, Derry S, Carr DB, Aldington D, Cole P, and Moore RA (2017) Morphine for chronic neuropathic pain in adults. Cochrane Database Syst Rev 5:CD011669.

Deakin JF and Dostrovsky JO (1978) Involvement of the periaqueductal grey matter and spinal 5-hydroxytryptaminergic pathways in morphine analgesia: effets of lesions and 5-hydroxytryptamine depletion. Br J Pharmacol 63:159-165.

DeSantana JM, da Cruz KM, and Sluka KA (2013) Animal models of fibromyalgia. Arthritis Res Ther 15:222.

Ebert B, Andersen S, and Krogsgaard-Larsen P (1995) Ketobemidone, methadone and pethidine are non-competitive N-methyl-D-aspartate (NMDA) antagonists in the rat cortex and spinal cord. Neurosci Lett 187:165-168.

Feng B, Morley RM, Jane DE, and Monaghan DT (2005) The effect of competitive antagonist chain length on NMDA receptor subunit selectivity. Neuropharmacology 48:354-359.

Ferdousi M and Finn DP (2018) Stress-induced modulation of pain: role of the endogenous opioid system. Prog Brain Res 239:121-177.

Fields HL, Vanegas H, Hentall ID, and Zorman G (1983) Evidence that disinhibition of brain stem neurones contributes to morphine analgesia. Nature 306:684-686.

Goldenberg DL, Clauw DJ, Palmer RE, and Clair AG (2016) Opioid use in fibromyalgia: a cautionary tale. Mayo Clin Proc 91:640-648. 
Gorman AL, Elliott KJ, and Inturrisi CE (1997) The d- and l-isomers of methadone bind to the non-competitive site on the N-methyl-D-aspartate (NMDA) receptor in rat forebrain and spinal cord. Neurosci Lett 223:5-8.

Guissouma H, Froidevaux MS, Hassani Z, and Demeneix BA (2006) In vivo siRNA delivery to the mouse hypothalamus confirms distinct roles of TR beta isoforms in regulating TRH transcription. Neurosci Lett 406:240-243.

Hargreaves K, Dubner R, Brown F, Flores C, and Joris J (1988) A new and sensitive method for measuring thermal nociception in cutaneous hyperalgesia. Pain 32 $77-88$

Harris RE, Clauw DJ, Scott DJ, McLean SA, Gracely RH, and Zubieta JK (2007) Decreased central mu-opioid receptor availability in fibromyalgia. $J$ Neurosci 27 : 10000-10006.

Häuser W, Schug S, and Furlan $\mathrm{AD}$ (2017) The opioid epidemic and national guidelines for opioid therapy for chronic noncancer pain: a perspective from different continents. Pain Rep 2:e599.

Inoue M, Mishina M, and Ueda H (2003) Locus-specific rescue of GluRepsilon1 NMDA receptors in mutant mice identifies the brain regions important for morphine tolerance and dependence. $J$ Neurosci 23:6529-6536.

Inoue M, Rashid MH, Fujita R, Contos JJ, Chun J, and Ueda H (2004) Initiation of neuropathic pain requires lysophosphatidic acid receptor signaling. Nat Med 10: $712-718$.

Jennings EM, Okine BN, Roche M, and Finn DP (2014) Stress-induced hyperalgesia. Prog Neurobiol 121:1-18.

Khasar SG, Green PG, and Levine JD (2005) Repeated sound stress enhances inflammatory pain in the rat. Pain 116:79-86.

Khasar SG, Green PG, Miao FJ, and Levine JD (2003) Vagal modulation of nociception is mediated by adrenomedullary epinephrine in the rat. Eur J Neurosci 17 909-915.

Kilkenny C, Browne W, Cuthill IC, Emerson M, and Altman DG; NC3Rs Reporting Guidelines Working Group (2010) Animal research: reporting in vivo experiments: the ARRIVE guidelines. Br J Pharmacol 160:1577-1579.

Koshimizu TA, Honda K, Nagaoka-Uozumi S, Ichimura A, Kimura I, Nakaya M, Sakai N, Shibata K, Ushijima K, Fujimura A, et al. (2018) Complex formation between the vasopressin $1 \mathrm{~b}$ receptor, $\beta$-arrestin-2, and the $\mu$-opioid receptor underlies morphine tolerance. Nat Neurosci 21:820-833.

Martínez-Navarro M, Maldonado R, and Baños JE (2019) Why mu-opioid agonists have less analgesic efficacy in neuropathic pain? Eur J Pain 23:435-454.

Matsumoto M, Xie W, Ma L, and Ueda H (2008) Pharmacological switch in Abetafiber stimulation-induced spinal transmission in mice with partial sciatic nerve injury. Mol Pain 4:25.

Matsushita Y, Omotuyi IO, Mukae T, and Ueda H (2013) Microglia activation precedes the anti-opioid BDNF and NMDA receptor mechanisms underlying morphine analgesic tolerance. Curr Pharm Des 19:7355-7361.

McGrath JC, Drummond GB, McLachlan EM, Kilkenny C, and Wainwright CL (2010) Guidelines for reporting experiments involving animals: the ARRIVE guidelines. Br J Pharmacol 160:1573-1576.

McGrath JC and Lilley E (2015) Implementing guidelines on reporting research using animals (ARRIVE etc.): new requirements for publication in BJP. $\mathrm{Br}$ $J$ Pharmacol 172:3189-3193.

Millan MJ (2002) Descending control of pain. Prog Neurobiol 66:355-474

Mukae T, Fujita W, and Ueda H (2016) P-glycoprotein inhibitors improve effective dose and time of pregabalin to inhibit intermittent cold stress-induced central pain. J Pharmacol Sci 131:64-67.

Mutel V, Buchy D, Klingelschmidt A, Messer J, Bleuel Z, Kemp JA, and Richards JG (1998) In vitro binding properties in rat brain of $[3 \mathrm{H}] \mathrm{Ro} 25-6981$, a potent and selective antagonist of NMDA receptors containing NR2B subunits. $J$ Neurochem 70:2147-2155.

Nagai J, Kurokawa M, Takeshima H, Kieffer BL, and Ueda H (2007) Circadiandependent learning and memory enhancement in nociceptin receptor-deficient mice with a novel KUROBOX apparatus using stress-free positive cue task $J$ Pharmacol Exp Ther 321:195-201.

Nagakura Y, Oe T, Aoki T, and Matsuoka N (2009) Biogenic amine depletion causes chronic muscular pain and tactile allodynia accompanied by depression: a putative animal model of fibromyalgia. Pain 146:26-33.

Niethammer M, Kim E, and Sheng M (1996) Interaction between the C terminus of NMDA receptor subunits and multiple members of the PSD-95 family of membrane-associated guanylate kinases. J Neurosci 16:2157-2163.

Nishiyori M, Nagai J, Nakazawa T, and Ueda H (2010) Absence of morphine analgesia and its underlying descending serotonergic activation in an experimental mouse model of fibromyalgia. Neurosci Lett 472:184-187.

Nishiyori M, Uchida H, Nagai J, Araki K, Mukae T, Kishioka S, and Ueda H (2011) Permanent relief from intermittent cold stress-induced fibromyalgia-like abnormal pain by repeated intrathecal administration of antidepressants. Mol Pain 7:69.
Nishiyori M and Ueda H (2008) Prolonged gabapentin analgesia in an experimental mouse model of fibromyalgia. Mol Pain 4:52.

Paxions G and Franklin KBJ (2001) The Mouse Brain in Stereotaxic Coordinates, 2nd ed, Academic Press, San Diego, CA.

Rashid MH, Inoue M, Toda K, and Ueda H (2004) Loss of peripheral morphine analgesia contributes to the reduced effectiveness of systemic morphine in neuropathic pain. J Pharmacol Exp Ther 309:380-387.

Sakimura K, Kutsuwada T, Ito I, Manabe T, Takayama C, Kushiya E, Yagi T, Aizawa S, Inoue Y, Sugiyama H, et al. (1995) Reduced hippocampal LTP and spatial learning in mice lacking NMDA receptor epsilon 1 subunit. Nature 373:151-155.

Sato $\mathrm{M}$ and Takagi H (1971) Enhancement by morphine of the central descending inhibitory influence on spinal sensory transmission. Eur J Pharmacol 14:60-65.

Schrepf A, Harper DE, Harte SE, Wang H, Ichesco E, Hampson JP, Zubieta JK, Clauw DJ, and Harris RE (2016) Endogenous opioidergic dysregulation of pain in fibromyalgia: a PET and fMRI study. Pain 157:2217-2225.

Sluka KA, Kalra A, and Moore SA (2001) Unilateral intramuscular injections of acidic saline produce a bilateral, long-lasting hyperalgesia. Muscle Nerve 24:37-46 Stanic J, Carta M, Eberini I, Pelucchi S, Marcello E, Genazzani AA, Racca C, Mulle C, Di Luca M, and Gardoni F (2015) Rabphilin 3A retains NMDA receptors at synaptic sites through interaction with GluN2A/PSD-95 complex. Nat Commun 6: 10181.

Takagi H, Satoh M, Akaike A, Shibata T, Yajima H, and Ogawa H (1978) Analgesia by enkephalins injected into the nucleus reticularis gigantocellularis of rat medulla oblongata. Eur J Pharmacol 49:113-116.

Tanaka K, Furuyashiki T, Kitaoka S, Senzai Y, Imoto Y, Segi-Nishida E, Deguchi Y, Breyer RM, Breyer MD, and Narumiya S (2012) Prostaglandin E2-mediated attenuation of mesocortical dopaminergic pathway is critical for susceptibility to repeated social defeat stress in mice. J Neurosci 32:4319-4329.

Taylor CP, Traynelis SF, Siffert J, Pope LE, and Matsumoto RR (2016) Pharmacology of dextromethorphan: relevance to dextromethorphan/quinidine (Nuedexta@) clinical use. Pharmacol Ther 164:170-182.

Uchida H, Ma L, and Ueda H (2010) Epigenetic gene silencing underlies C-fiber dysfunctions in neuropathic pain. $J$ Neurosci 30:4806-4814.

Uchida H, Nagai J, and Ueda H (2014) Lysophosphatidic acid and its receptors LPA1 and LPA3 mediate paclitaxel-induced neuropathic pain in mice. Mol Pain 10:71.

Ueda H (2017) Lysophosphatidic acid signaling is the definitive mechanism underlying neuropathic pain. Pain 158 (Suppl 1):S55-S65

Ueda H and Neyama H (2017) LPA1 receptor involvement in fibromyalgia-like pain induced by intermittent psychological stress, empathy. Neurobiol Pain 1:16-25.

Ueda H, Neyama H, Nagai J, Matsushita Y, Tsukahara T, and Tsukahara R (2018a) Involvement of lysophosphatidic acid-induced astrocyte activation underlying the maintenance of partial sciatic nerve injury-induced neuropathic pain. Pain 159: $2170-2178$

Ueda H, Neyama H, Sasaki K, Miyama C, and Iwamoto R (2018b) Lysophosphatidic acid $\mathrm{LPA}_{1}$ and $\mathrm{LPA}_{3}$ receptors play roles in the maintenance of late tissue plasminogen activator-induced central poststroke pain in mice. Neurobiol Pain 5: 100020.

van Weering HR, Boddeke HW, Vinet J, Brouwer N, de Haas AH, van Rooijen N, Thomsen AR, and Biber KP (2011) CXCL10/CXCR3 signaling in glia cells differentially affects NMDA-induced cell death in CA and DG neurons of the mouse hippocampus. Hippocampus 21:220-232.

Wang W, Peng Y, Yang H, Bu H, Guo G, Liu D, Shu B, Tian X, Luo A, Zhang X, et al. (2017) Potential role of CXCL10/CXCR3 signaling in the development of morphine tolerance in periaqueductal gray. Neuropeptides 65:120-127.

Webster L (2013) Methadone Side Effects: Constipation, Respiratory Depression, Se dation, Sleep-Disordered Breathing, and the Endocrine System, Springer, New York.

Yamamoto J, Kawamata T, Niiyama Y, Omote K, and Namiki A (2008) Downregulation of $\mathrm{mu}$ opioid receptor expression within distinct subpopulations of dorsal root ganglion neurons in a murine model of bone cancer pain. Neuroscience 151:843-853.

Yokoyama T, Maeda Y, Audette KM, and Sluka KA (2007) Pregabalin reduces muscle and cutaneous hyperalgesia in two models of chronic muscle pain in rats. J Pain 8: $422-429$.

Zimmermann M (1983) Ethical guidelines for investigations of experimental pain in conscious animals. Pain 16:109-110.

Address correspondence to: Hiroshi Ueda, Department of Molecular Pharmacology, Kyoto University Graduate School of Pharmaceutical Sciences, Yoshida Shimoadachi-cho, Sakyo-ku, Kyoto 606-8501, Japan. E-mail: ueda.hiroshi.8e@kyoto-u.ac.jp 